\title{
Total quantity of caesium radioisotopes in fish in the Fukushima-Ken Exclusive Economic Zone, Japan, in November 2012
}

\author{
Hidemitsu KATSURA ${ }^{1,2}$ \\ ${ }^{1}$ The United Graduate School of Agricultural Science, Tokyo University of Agriculture and Technology, Saiwai-Cho 3- \\ Chome, Fuchu-Shi, Tokyo 183-8509, Japan. \\ ${ }^{2}$ Department of Ocean Sciences, Faculty of Marine Science, Tokyo University of Marine Science and Technology, \\ Konan 4-Chome, Minato-Ku, Tokyo 108-8477, Japan.
}

Accepted 9 July, 2013

\begin{abstract}
The Tokyo Electric Power Company Fukushima Dai-ichi nuclear power plant in Fukushima-Ken (Prefecture), Japan, was destroyed in March 2011 due to a massive earthquake (magnitude 9) centred offshore to the northeast of Honshu Island and the subsequent historic tsunami on 11 March 2011. Due to nuclear meltdown, damage to the buildings housing the reactors by hydrogen explosions, and the contamination of cooling water from the reactor cores, huge quantities of radioisotopes were emitted to the atmosphere and to the adjacent seawater. Fishing is currently prohibited off the coast of Fukushima-Ken because intermittent surveys have found that the majority of fishery products still contain radioisotope levels exceeding the Japanese Standard Value. However, the total amount of caesium radioisotopes in fish biomass in the Fukushima-Ken Exclusive Economic Zone (EEZ) has not yet been reported. The Tokyo University of Marine Science and Technology has measured radioisotope levels in fishery species off Iwaki-Shi (City), Fukushima-Ken (located south of the destroyed nuclear power plant), and these data can be used to estimate the total fish biomass and the quantity of caesium radioisotopes in fish in the Fukushima-Ken EEZ. On 22 to 23 November 2012, the estimated total fish biomass in the Fukushima-Ken EEZ was $91,150,218.79 \mathrm{~kg}$ and the estimated total quantities of Cs-134 and $\mathrm{Cs}-137$ in fish were $4,879,259,657 \mathrm{~Bq}$ and $8,381,002,727 \mathrm{~Bq}$, respectively.
\end{abstract}

Key words: Nuclear power plant, Exclusive Economic Zone (EEZ), radioisotope.

\section{INTRODUCTION}

The Tokyo Electric Power Company Fukushima Dai-ichi nuclear power plant, located in Futaba-Gun (County), Fukushima-Ken (Prefecture), Japan, was destroyed in March 2011 due to a massive earthquake (magnitude 9) centred offshore to the northeast of Honshu Island and the subsequent historic tsunami on 11 March, 2011. Due to the resulting nuclear meltdown, damage to the buildings housing the reactors by hydrogen explosions, and the contamination of cooling water from the reactor cores, huge quantities of radioisotopes were emitted to the atmosphere and to the adjacent seawater. Fishing is currently prohibited off the coast of Fukushima-Ken because intermittent surveys have found that the majority of fishery products still contain radioisotope levels exceeding the Japanese Standard Value. Therefore, the Japanese government has measured radioisotope levels in fishery species not only offshore near the destroyed reactors but also throughout the 
Japanese Exclusive Economic Zone (EEZ). The Japanese government has regularly released a subset of these measurements to the public, for example, via the internet (Japanese Governmental Off. Bull. On-Line, 2012; Ministry of Education, C., S., S. \& T., 2011; NEMOTO et al., 2012; Ohnishi, 2012). To date, however, the total quantity of fishery resources and the estimated total quantity of caesium radioisotopes in fish biomass in the Fukushima-Ken EEZ after 11 March, 2011 have not been reported (Wan et al., 2012). This information is important not only to Japanese residents but also to residents of other countries. These data would make it possible to calculate overall contamination levels and to predict future trends. On 22 to 23 November, 2012, the Tokyo University of Marine Science and Technology performed an independent sampling measure radioisotope levels in fishery species off Iwaki-Shi (City), Fukushima-Ken (located south of the destroyed nuclear power plant). These data included detailed measurements of individual fish, such as weight, sex, length, and collection locality. Thus, this information could be used to estimate the fish biomass and the total quantity of caesium radioisotopes in fish in the Fukushima-Ken EEZ during November 2012. These estimates are reported here.

\section{MATERIALS AND METHODS}

Trawl (dragnet) fishing was performed on 22 November, 2012 off Yotsukura and Ena, Iwaki-Shi, Fukushima-Ken, Japan to sample the fish in the region. The total fishing areas were 9,450 and 9,775 square meter off Yotsukura and Ena, respectively (Atkins and Warren, 1953). Gill-net fishing was performed on 22 to 23 November, 2012 in the same locations. The total fishing areas were 28,900 and 50,141 square meter off Yotsukura and Ena, respectively (Buscaino et al., 2009). Subsequently, the edible portions of the fish were minced, and U-8 containers $(100 \mathrm{~mL})$ were filled with the minced fish flesh. The concentrations of the radioisotopes $\mathrm{Cs}-134$ and $\mathrm{Cs}-137$ in the fish biomass were measured by IDEA Consultants Inc. (Tokyo, Japan) using a germanium semiconductor detector (Seiko EG\&G Co. model GEM20-70) (Inazu et al., 2011; Minatani et al., 2012).

\section{RESULTS AND DISCUSSION}

(1) The total fish-collection area on 22 to 23 November, 2012 was as follows (Table 1):

$9,450+28,900+9,775+50,141=98,266$ square meter.

The total area of the Fukushima-Ken EEZ is $33,300,000,000$ square meter.

\section{$(33,300,000,000 / 98,266)=338,876.1118$}

Thus, the estimated biomass of all fish species in the Fukushima-Ken EEZ in November 2012 can be calculated by multiplying $338,876.1118$ times the total weight of the collected fish, and the total quantity of caesium radioisotopes (Cs-134 and Cs-137) in the fish biomass can be estimated using the following equations:

$[E T F W]=$ Estimated total fish weight in the FukushimaKen EEZ [kg]

[ACS134] $=$ Average Cs-134 level in fish biomass in the Fukushima-Ken EEZ in November 2012 [Bq/kg]

[Estimated Total Cs-134 in Fish] = Estimated total quantity of Cs-134 in fish biomass in the Fukushima-Ken EEZ in November 2012 [Bq]

[ACS137] = Average Cs-137 level in fish biomass in the Fukushima-Ken EEZ in November 2012 [Bq/kg]

[Estimated Total Cs-137 in Fish] = Estimated total quantity of Cs-137 in fish biomass in the Fukushima-Ken EEZ in November 2012 [Bq]

[Estimated Total Cs-134 in Fish $]=[$ ETFW] X [ACS134] [Estimated Total Cs-137 in Fish $]=[\mathrm{ETFW}] \mathrm{X}$ [ACS137]

Table 2 shows the resulting estimates. The estimated total fish weight in the Fukushima-Ken EEZ on 22 to 23 November, 2012 was $91,150,218.79 \mathrm{~kg}$; the estimated total quantity of Cs-134 in all fish in the Fukushima-Ken EEZ was 4,879,259,657 Bq; and the estimated total quantity of Cs-137 in all fish in the Fukushima-Ken EEZ was $8,381,002,727 \mathrm{~Bq}$.

[2] Some scholars and other professionals do not agree with this estimation method because the range of a particular fish species depends on environmental conditions, and they believe that it is not meaningful to extrapolate from fish sampled off the coast of FukushimaKen to the total area of the Fukushima-Ken EEZ. On the other hand, not only Japanese residents but also residents of other countries, especially those living in Pacific coastal areas, are strongly interested in the total quantity of radioisotopes in fish biomass in the Fukushima-Ken EEZ. This information is critical to an overall perspective and future prediction of radioactive contamination in Pacific fisheries. One way to address this contradiction is to examine historical accidents at nuclear power plants, such as those at the Three Mile Island Nuclear Generating Station in Pennsylvania, U.S.A. (1979) and at the Chernobyl Nuclear Power Plant in Ukraine, former U.S.S.R. (1986) (Macdonald et al., 1987; Asmolov et al. 1987). However, these accidents did not occur along marine coastlines. On the other hand, governments conducted frequent atmospheric nuclear tests prior to the effective date of the Treaty Banning Nuclear Weapon Tests in the Atmosphere, in Outer Space and Under Water [often abbreviated as the Partial Test Ban Treaty (PTBT), Limited Test Ban Treaty (LTBT), or Nuclear Test Ban Treaty (NTBT)] in October 1963 (Scherb and Voigt, 2011; Lown and Pastore, 1985). Some atmospheric nuclear tests were conducted in the ocean, and reported estimates of the total Amounts 
Table 1. Total fish-collection area on 22 to 23 November, 2012.

\begin{tabular}{|c|c|c|c|c|c|c|c|}
\hline Location & YOTSUKURA & YOTSUKURA & ENA & ENA (Land Side) & & & \\
\hline Way of sampling & $\begin{array}{l}\text { Trawl (Net) [Dragneta } \\
\text { Trawlnet] (HIKIAMI) }\end{array}$ & $\begin{array}{l}\text { Gill Net } \\
\text { (SASHIAMI) }\end{array}$ & $\begin{array}{l}\text { Trawl (Net) [Dragneta } \\
\text { Trawlnet] (HIKIAMI) (2 Times) }\end{array}$ & Gill Net (SASHIAMI) & & & \\
\hline $\begin{array}{l}\text { Sampling date for weight } \\
\text { of fishes }\end{array}$ & 22- NOV-2012 & 22 to 23 NOV-2012 & 22- NOV-2012 & 22 to 23 NOV-2012 & $\begin{array}{l}\text { Total fish collecting area in } 22 \\
\text { to } 23 \text { NOV-2012 }\left[\mathrm{m}^{2}\right]=[\text { TFCA }]\end{array}$ & $\begin{array}{l}\text { Total area of FUKUSHIMA- } \\
\text { KEN EEZ [m²] = [TAFEEZ] }\end{array}$ & $\begin{array}{l}{[\text { TAFEEZ] } /} \\
{[\text { TFCA] }}\end{array}$ \\
\hline Area [m²] & 9450 & 28900 & 9775 & 50141 & 98266 & 33300000000 & 338876.1118 \\
\hline
\end{tabular}

Table 2. Amount of fishery resources and the estimated total amount of cesium radioisotopes in fish bodies in offshore Fukushima-Ken, Japan in November 2012.

\begin{tabular}{|c|c|c|c|c|c|c|c|c|c|c|c|c|c|c|c|c|c|c|c|c|c|}
\hline Scientific Name & $\begin{array}{l}\text { Japanese } \\
\text { Name }\end{array}$ & $\begin{array}{c}\text { Weight [g] } \\
\text { at } \\
\text { YoTSUKUR } \\
\text { A by Trawl } \\
\text { (Net) }\end{array}$ & $\begin{array}{l}\text { Weight \% at } \\
\text { YOTSUKURA } \\
\text { by Trawl (Net) }\end{array}$ & $\begin{array}{c}\text { Weight [g] } \\
\text { at } \\
\text { YoTSUKU } \\
\text { RA by Gill } \\
\text { Net }\end{array}$ & $\begin{array}{c}\text { Weight \% at } \\
\text { YOTSUKURA } \\
\text { by Gill Net }\end{array}$ & $\begin{array}{l}\text { Weight [g] } \\
\text { at ENA by } \\
\text { Trawl (Net) } \\
\text { (2 Times) }\end{array}$ & $\begin{array}{l}\text { Weight \% at } \\
\text { ENA by Trawl } \\
\text { (Net) }(2 \\
\text { Times) }\end{array}$ & $\begin{array}{l}\text { Weight [g] } \\
\text { at ENA } \\
\text { (Land } \\
\text { Side) by } \\
\text { Gill Net }\end{array}$ & $\begin{array}{l}\text { Weight \% at } \\
\text { ENA (Land } \\
\text { Side) by Gill } \\
\text { Net }\end{array}$ & $\begin{array}{c}\text { Weight [g] } \\
\text { at ENA } \\
\text { (Offshore } \\
\text { Side) by } \\
\text { Gill Net }\end{array}$ & $\begin{array}{l}\text { Weight \% at } \\
\text { ENA (Offshore } \\
\text { Side) by Gill } \\
\text { Net }\end{array}$ & $\begin{array}{l}\text { Grand } \\
\text { Fish } \\
\text { Weight } \\
\text { Toat [g] }\end{array}$ & $\begin{array}{l}\text { Grand Fish } \\
\text { Weight \% }\end{array}$ & $\begin{array}{l}\text { Estimated Total } \\
\text { Fish Weight in } \\
\text { FUKUSHIMA- } \\
\text { KEN EEZ [g] }\end{array}$ & $\begin{array}{l}\text { Estimated } \\
\text { Total Fish } \\
\text { Weight in } \\
\text { FUKUSHIMA- } \\
\text { KEN EEZ [kg] }\end{array}$ & $\begin{array}{l}\text { Average Cs- } \\
134 \text { in Fish in } \\
\text { NOV-2012 in } \\
\text { FUKUSHIMA- } \\
\text { KEN EEZ } \\
\text { [becquerel]/[k } \\
\text { g] }\end{array}$ & $\begin{array}{c}\text { Estimated Total Cs- } \\
134 \text { in Fish Body in } \\
\text { NOV-2012 in } \\
\text { FUKUSHIMA-KEN } \\
\text { EEZ [becquerel] = } \\
\text { k Estimated Total Cs- } \\
134 \text { in Fish] } \\
\end{array}$ & $\begin{array}{c}\text { ([Estimated } \\
\text { Cs-134 in } \\
\text { Fish] } / \\
\text { [Estimated } \\
\text { Total Cs-134 } \\
\text { - in Fish]) } x \\
100[\%] \\
\end{array}$ & $\begin{array}{c}\text { Average Cs- } \\
137 \text { in Fish in } \\
\text { in NOV-2012 } \\
\text { FUKUSHIMA- } \\
\text { KEN EEZ } \\
\text { [becquerel]/[k } \\
\text { g] } \\
\end{array}$ & $\begin{array}{c}\text { Estimated Total Cs- } \\
137 \text { in Fish Body in } \\
\text { NOV-2012 in } \\
\text { FUKUSHIMA-KEN } \\
\text { EEZ [becquerel] = } \\
\text { [Estimated Total Cs- } \\
137 \text { in Fish] }\end{array}$ & $\begin{array}{l}\text { ([Estimated Cs- } \\
137 \text { in Fish] } / \\
\text { [Estimated Total } \\
\text { Cs-137 in Fish]) } \\
\quad \times 100[\%]\end{array}$ \\
\hline $\begin{array}{l}\text { sAcanthopagrus } \\
\text { schlegeli }\end{array}$ & KURODAI & 2000 & 1.456261195 & 0 & 0 & 0 & 0 & 0 & 0 & 0 & 0 & 2000 & 0.743555235 & $5 \quad 677752223.6$ & 677752.2236 & 0 & 0 & 0 & 0 & 0 & 0 \\
\hline $\begin{array}{l}\text { Chelidonichthys } \\
\text { spinosus }\end{array}$ & HOUBOU & 0 & 0 & 1170 & 3.549757282 & 0 & 0 & 0 & 0 & 0 & 0 & 1170 & 0.434979812 & $2 \quad 396485050.8$ & 396485.0508 & 7.015 & 2781342.631 & 0.057003374 & 10.085 & 3998551.737 & 0.047709706 \\
\hline $\begin{array}{l}\text { Clupea pallasiii, } \\
\text { Vakenciennes }\end{array}$ & iii NISHIN & 23 & 0.016747004 & 0 & 0 & 0 & 0 & 0 & 0 & 0 & 0 & 23 & 0.008550885 & $5 \quad 7794150.571$ & 7794.150571 & 0 & 0 & 0 & 0 & 0 & 0 \\
\hline $\begin{array}{l}\text { Cynoglossus } \\
\text { joyneri }\end{array}$ & $\begin{array}{l}\text { AKASHITA } \\
\text { BIRAME }\end{array}$ & 600 & 0.436878359 & 0 & 0 & 1810 & 4.815110402 & 300 & 2.284843869 & 0 & 0 & 2710 & 1.007517343 & $\begin{array}{l}3 \\
\end{array}$ & 918354.2629 & 14.1 & 12948795.11 & 0.265384423 & 22.25 & 20433382.35 & 0.243805938 \\
\hline $\begin{array}{l}\text { Ditrema } \\
\text { temminkii }\end{array}$ & $\begin{array}{l}\text { UMI } \\
\text { TANAGO }\end{array}$ & 0 & 0 & 0 & 0 & 0 & & 210 & 1.599390708 & 2960 & 6.171809842 & 3170 & 1.178535047 & $7 \quad 1074237274$ & 1074237.274 & 12.445 & 13368882.88 & 0.273994086 & 22.3 & 23955491.22 & 0.285830849 \\
\hline $\begin{array}{l}\text { Engraulis } \\
\text { japonica }\end{array}$ & $\begin{array}{l}\text { KATAKUCH } \\
\text { IIWASHI }\end{array}$ & 88 & 0.064075493 & 0 & 0 & 0 & 0 & 0 & 0 & 0 & 0 & 88 & 0.03271643 & 29821097.84 & 29821.09784 & 0 & 0 & 0 & 0 & 0 & 0 \\
\hline $\begin{array}{l}\text { Hexagrammos } \\
\text { otakii }\end{array}$ & AINAME & 0 & 0 & 2130 & 6.462378641 & & 0 & 1060 & 8.073115004 & 1810 & 3.773978315 & 5000 & 1.858888088 & $8 \quad 1694380559$ & 1694380.559 & 52.19230769 & 88433631.48 & 1.812439544 & 88.01538462 & 149131556.6 & 1.779399929 \\
\hline $\begin{array}{l}\text { Hexagrammos } \\
\text { stelleri }\end{array}$ & $\begin{array}{l}\text { EZO ISO } \\
\text { AINAME }\end{array}$ & 0 & 0 & 0 & 0 & 0 & 0 & 330 & 2.513328256 & 940 & 1.959966639 & 1270 & 0.472157574 & $4 \quad 430372662$ & 430372.662 & 21.9 & 9425161.297 & 0.193167857 & 37.36666667 & 16081591.8 & 0.191881477 \\
\hline $\begin{array}{l}\text { Kareius } \\
\text { bicoloratus }\end{array}$ & ISHI GAREI & 0 & 0 & 750 & 2.275485437 & 920 & 2.447459431 & 0 & 0 & 0 & 0 & 1670 & 0.620868621 & 1565923106.7 & 565923.1067 & 130 & 73570003.87 & 1.507810796 & 213 & 120541621.7 & 1.438272074 \\
\hline $\begin{array}{l}\text { Lateolabrax } \\
\text { japonicus }\end{array}$ & SUZUKI & 0 & 0 & 0 & 0 & 1300 & 3.458366587 & 0 & 0 & 0 & 0 & 1300 & 0.483310903 & 3440538945.3 & 440538.9453 & 53.9 & 23745049.15 & 0.486652706 & 98.6 & 43437140.01 & 0.518280944 \\
\hline $\begin{array}{l}\text { Lepidotrigla } \\
\text { microptena } \\
\text { Gunther }\end{array}$ & $\begin{array}{l}\text { KANA } \\
\text { GASHIRA }\end{array}$ & 280 & 0.203876567 & 0 & 0 & 0 & 0 & 0 & 0 & 0 & 0 & 280 & 0.104097733 & $\begin{array}{ll}3 & 94885311.3\end{array}$ & 94885.3113 & 0 & 0 & 0 & 0 & 0 & 0 \\
\hline Liparis tanakai & KUSAUO & 310 & 0.225720485 & 0 & 0 & 0 & 0 & 0 & 0 & 0 & 0 & 310 & 0.115251061 & $1 \quad 105051594.7$ & 105051.5947 & 0 & 0 & 0 & 0 & 0 & 0 \\
\hline $\begin{array}{l}\text { Mlicrostomus } \\
\text { achn }\end{array}$ & $\begin{array}{l}\text { BABA } \\
\text { GAREI }\end{array}$ & 0 & 0 & 2600 & 7.888349515 & 0 & 0 & 0 & 0 & 850 & 1.772310259 & 3450 & 1.28263278 & 1169122586 & 1169122.586 & 107.8333333 & 126070385.5 & 2.583801526 & 187.3333333 & 219015631 & 2.61323899 \\
\hline Mustelus manazo & $\begin{array}{l}\text { HOSHI } \\
\text { OZAME }\end{array}$ & 50500 & 36.77059517 & 0 & 0 & 11100 & 29.52913009 & 0 & 0 & 0 & 0 & 61600 & 22.90150124 & $4 \quad 20874768486$ & 20874768.49 & 0 & 0 & 0 & 0 & 0 & 0 \\
\hline Nibea mitsukurii & NIBE & 0 & 0 & 0 & 0 & 0 & 0 & 1140 & 8.682406702 & 1040 & 2.168473728 & 2180 & 0.810475206 & $\begin{array}{l}6 \quad 738749923.7 \\
\end{array}$ & 738749.9237 & 16.66 & 12307573.73 & 0.252242647 & 29.9 & 22088622.72 & 0.263555847 \\
\hline Okamejei kenojei & $\begin{array}{l}\text { KOMON } \\
\text { KASUBE }\end{array}$ & 56900 & 41.430631 & 1390 & 4.21723301 & 13480 & 35.86060122 & 0 & 0 & 0 & 0 & 71770 & 26.68247961 & 124321138542 & 24321138.54 & 98.95 & 2406576659 & 49.32257817 & 173.95 & 4230662049 & 50.47918713 \\
\hline $\begin{array}{l}\text { Oncorhynchus } \\
\text { keta }\end{array}$ & SAKE & 0 & 0 & 0 & 0 & 0 & 0 & 0 & 0 & 500 & 1.042535446 & 500 & 0.185888809 & $9 \quad 169438055.9$ & 169438.0559 & 0 & 0 & 0 & 0 & 0 & 0 \\
\hline $\begin{array}{l}\text { Oplegnathus } \\
\text { punctatus }\end{array}$ & $\begin{array}{l}\text { ISHIGAKI } \\
\text { DAI }\end{array}$ & 0 & 0 & 640 & 1.941747573 & 0 & 0 & 0 & 0 & 0 & 0 & 640 & 0.237937675 & $5 \quad 216880711.5$ & 216880.7115 & 0 & 0 & 0 & 0 & 0 & 0 \\
\hline
\end{tabular}


Table 2. Contd.

\begin{tabular}{|c|c|c|c|c|c|c|c|c|c|c|c|c|c|c|c|c|c|c|c|c|c|}
\hline Scientific Name & $\begin{array}{l}\text { Japanese } \\
\text { Name }\end{array}$ & $\begin{array}{c}\text { Weight [g] at } \\
\text { YOTSUURR } \\
\text { A by Trawl } \\
\text { (Net) }\end{array}$ & $\begin{array}{l}\text { Weight \% at } \\
\text { YOTSUKURA } \\
\text { by Trawl (Net) }\end{array}$ & $\begin{array}{l}\text { Weight } \\
\text { [g] at } \\
\text { YOTSUK } \\
\text { URA by } \\
\text { Gill Net }\end{array}$ & $\begin{array}{l}\text { Weight \% at } \\
\text { YOTSUKURA } \\
\text { by Gill Net }\end{array}$ & $\begin{array}{l}\text { Weight [g] } \\
\text { at ENA by } \\
\text { Trawl (Net) } \\
\text { (2 Times) }\end{array}$ & $\begin{array}{l}\text { Weight } \% \text { at } \\
\text { ENA by Trawl } \\
\text { (Net) }(2 \\
\text { Times) }\end{array}$ & $\begin{array}{l}\text { Weight [g] } \\
\text { at ENA } \\
\text { (Land } \\
\text { Side) by } \\
\text { Gill Net }\end{array}$ & $\begin{array}{c}\text { Weight \% at } \\
\text { ENA (Land } \\
\text { Side) by Gill } \\
\text { Net }\end{array}$ & $\begin{array}{l}\text { Weight [g] } \\
\text { at ENA } \\
\text { (Offshore } \\
\text { Side) by } \\
\text { Gill Net }\end{array}$ & $\begin{array}{l}\text { Weight \% at } \\
\text { ENA (Offshore } \\
\text { Side) by Gill } \\
\text { Net }\end{array}$ & $\begin{array}{l}\text { Grand } \\
\text { Fish } \\
\text { Weight } \\
\text { Toatl [g] }\end{array}$ & $\begin{array}{l}\text { Grand Fish } \\
\text { Weight \% }\end{array}$ & $\begin{array}{l}\text { Estimated Total } \\
\text { Fish Weight in } \\
\text { FUKUSHIMA- } \\
\text { KEN EEZ [g] }\end{array}$ & $\begin{array}{c}\text { Estimated } \\
\text { Total Fish } \\
\text { Weight in } \\
\text { FUKUSHIMA- } \\
\text { KEN EEZ [kg] }\end{array}$ & $\begin{array}{c}\text { Average Cs-134 } \\
\text { in Fish in NOV- } \\
2012 \text { in } \\
\text { FUKUSHIMA- } \\
\text { KEN EEZ } \\
\text { [becquerel]][kg] }\end{array}$ & $\begin{array}{c}\text { Estimated Total } \\
\text { Cs-134 in Fish } \\
\text { Body in NOV-2012 } \\
\text { in FUKUSHIMA- } \\
\text { KEN EEZ } \\
\text { [becquere] = } \\
\text { [Estimated Total } \\
\text { Cs-134 in Fish] }\end{array}$ & $\begin{array}{l}\text { ([Estimated } \\
\text { Cs-134 in } \\
\text { Fish] } / \\
\text { [Estimated } \\
\text { Total Cs-134 } \\
\text { in Fish]) } \mathrm{x} \\
100[\%] \\
\end{array}$ & $\begin{array}{l}\text { Average Cs- } \\
137 \text { in Fish in } \\
\text { in NOV-2012 } \\
\text { FUKUSHIMA- } \\
\text { KEN EEZ } \\
\text { [becquerel]][kg [ } \\
\text { ] }\end{array}$ & $\begin{array}{c}\text { Estimated Total Cs- } \\
137 \text { in Fish Body in } \\
\text { NOV-2012 in } \\
\text { FUKUSHIMA-KEN } \\
\text { EEZ [becquerel] = } \\
\text { [Estimated Total Cs- } \\
137 \text { in Fish] }\end{array}$ & $\begin{array}{l}\text { ([Estimated Cs- } \\
137 \text { in Fish] } / \\
\text { [Estimated Total } \\
\text { Cs-137 in Fish]) } \\
\quad x 100[\%]\end{array}$ \\
\hline Pagrus major & MADAI & 0 & 0 & 0 & 0 & 0 & 0 & 0 & 0 & 290 & 0.604670559 & 290 & 0.107815509 & 98274072.42 & 98274.07242 & 0 & 0 & 0 & & 0 & 0 \\
\hline $\begin{array}{l}\text { Pagrus majorl } \\
\text { (Fry) }\end{array}$ & $\begin{array}{l}\text { orMADAl } \\
\text { (CHIGYO) }\end{array}$ & 322 & 0.234458052 & 0 & 0 & 0 & 0 & 0 & 0 & 0 & 0 & 322 & 0.119712393 & 109118108 & 109118.108 & 0 & 0 & 0 & 0 & 0 & 0 \\
\hline $\begin{array}{l}\text { Paralichthys } \\
\text { olivaceus }\end{array}$ & HIRAME & 1600 & 1.165008956 & 6280 & 19.05339806 & 5340 & 14.20590583 & 0 & 0 & 2500 & 5.212677231 & 15720 & 5.844344147 & 5327132477 & 5327132.477 & 14.98 & 79800444.51 & 1.635503132 & 25.74727273 & 137159132.7 & 1.636548003 \\
\hline $\begin{array}{l}\text { Paralichthys } \\
\text { olivaceus (Fry) }\end{array}$ & $\begin{array}{l}\text { HIRAME } \\
\text { (CHIGYO) }\end{array}$ & 34 & 0.02475644 & 0 & 0 & 0 & 0 & 0 & 0 & 0 & 0 & 34 & 0.012640439 & 11521787.8 & 11521.7878 & 0 & 0 & 0 & 0 & 0 & 0 \\
\hline $\begin{array}{l}\text { Platycephalus } \\
\text { sp.2 }\end{array}$ & MAGOCHI & 580 & 0.422315747 & 0 & 0 & 450 & 1.197126895 & 0 & 0 & 0 & 0 & 1030 & 0.382930946 & 349042395.1 & 349042.3951 & 0 & 0 & 0 & 0 & 0 & 0 \\
\hline $\begin{array}{l}\text { Platycephalus } \\
\text { sp.2 (fry) }\end{array}$ & $\begin{array}{l}\text { MAGOCHI } \\
\text { (CHIGYO) }\end{array}$ & 10 & 0.007281306 & 0 & 0 & 0 & 0 & 0 & 0 & 0 & 0 & 10 & 0.003717776 & 3388761.118 & 3388.761118 & 0 & 0 & 0 & 0 & 0 & 0 \\
\hline $\begin{array}{l}\text { Scyliorhinus } \\
\text { torazame }\end{array}$ & $\begin{array}{l}\text { TORA } \\
\text { ZAME }\end{array}$ & 5200 & 3.786279107 & 250 & 0.758495146 & 3190 & 8.486299548 & & 0 & 0 & 0 & 8640 & 3.212158615 & 2927889606 & 2927889.606 & 27.00583333 & 79070098.71 & 1.620534759 & 47.58 & 139308987.4 & 1.662199524 \\
\hline Sebastes cheni & $\begin{array}{l}\text { SHIRO } \\
\text { MEBARU }\end{array}$ & 0 & 0 & 200 & 0.606796117 & 0 & 0 & 8890 & 67.70753998 & 27760 & 57.88156797 & 36850 & 13.7000052 & 12487584719 & 12487584.72 & 129.0857143 & 1611968793 & 33.03715945 & 213.2857143 & 2663423427 & 31.77929316 \\
\hline Sebastes inermis & $\begin{array}{l}\text { AKA } \\
\text { s MEBARU }\end{array}$ & 0 & 0 & 0 & 0 & 0 & 0 & 0 & 0 & 1020 & 2.12677231 & 1020 & 0.37921317 & 345653634 & 345653.634 & 96.175 & 33243238.25 & 0.681317261 & 160.15 & 55356429.49 & 0.660498884 \\
\hline $\begin{array}{l}\text { Sebastes } \\
\text { pachycephalus } \\
\text { pachycephalus }\end{array}$ & MURASOI & 0 & 0 & 0 & 0 & 0 & 0 & 330 & 2.513328256 & 1760 & 3.669724771 & 2090 & 0.777015221 & 708251073.6 & 708251.0736 & 40.175 & 28453986.88 & 0.583161973 & 70.65 & 50037938.35 & 0.597039996 \\
\hline Sebastes vulpes & $\begin{array}{l}\text { KITSUNE } \\
\text { MEBARU }\end{array}$ & 0 & 0 & 1260 & 3.822815534 & 0 & 0 & 590 & 4.493526276 & 6530 & 13.61551293 & 8380 & 3.115496435 & 2839781817 & 2839781.817 & 32.4 & 92008930.86 & 1.885714992 & 59.068 & 167740232.3 & 2.001433931 \\
\hline $\begin{array}{l}\text { Sebastiscus } \\
\text { marmoratus }\end{array}$ & KASAGO & 0 & 0 & 0 & 0 & 0 & 0 & 280 & 2.132520944 & 0 & 0 & 280 & 0.104097733 & 94885311.3 & 94885.3113 & 38.3 & 3634107.423 & 0.074480714 & 67.1 & 6366804.388 & 0.075967096 \\
\hline $\begin{array}{l}\text { Seriola } \\
\text { quinqueradiata }\end{array}$ & BURI & 0 & 0 & 1670 & 5.066747573 & 0 & 0 & 0 & 0 & 0 & 0 & 1670 & 0.620868621 & 565923106.7 & 565923.1067 & 4.03 & 2280670.12 & 0.046742135 & 5.09 & 2880548.613 & 0.034369976 \\
\hline $\begin{array}{l}\text { Takifugu } \\
\text { poecilonotus }\end{array}$ & $\begin{array}{l}\text { KOMON } \\
\text { FUGU }\end{array}$ & 70 & 0.050969142 & 0 & 0 & 0 & 0 & 0 & 0 & 0 & 0 & 70 & 0.026024433 & 23721327.82 & 23721.32782 & 0 & 0 & 0 & 0 & 0 & 0 \\
\hline Takifugu rubipes & $\begin{array}{l}\text { TORA } \\
\text { FUGU }\end{array}$ & 1610 & 1.172290262 & 0 & 0 & 0 & 0 & 0 & 0 & 0 & 0 & 1610 & 0.598561964 & 545590540 & 545590.54 & 83.9 & 45775046.3 & 0.938155571 & 135 & 73654722.89 & 0.878829483 \\
\hline Takifugu snyderi & $\begin{array}{l}\text { SHOUSAI } \\
\text { FUGU }\end{array}$ & 16200 & 11.79571568 & 0 & 0 & 0 & 0 & 0 & 0 & 0 & 0 & 16200 & 6.022797404 & 5489793011 & 5489793.011 & 0 & 0 & 0 & 0 & 0 & 0 \\
\hline $\begin{array}{l}\text { Takifugu } \\
\text { stictonotus }\end{array}$ & $\begin{array}{l}\text { GOMA } \\
\text { FUGU }\end{array}$ & 120 & 0.087375672 & 0 & 0 & 0 & 0 & 0 & 0 & 0 & 0 & 120 & 0.044613314 & 40665133.41 & 40665.13341 & 0 & 0 & 0 & 0 & 0 & 0 \\
\hline $\begin{array}{l}\text { Trachurus } \\
\text { japonicus }\end{array}$ & MAAJ & 887 & 0.64585184 & 0 & 0 & 0 & 0 & 0 & 0 & 0 & 0 & 887 & 0.329766747 & 300583111.1 & 300583.1111 & 0 & 0 & 0 & 0 & 0 & 0 \\
\hline Triakis scyllium & $\begin{array}{l}\text { DOCHI } \\
\text { ZAME }\end{array}$ & 0 & 0 & 14620 & 44.35679612 & 0 & 0 & 0 & 0 & 0 & 0 & 14620 & 5.435388768 & 4954368754 & 4954368.754 & 27.00583333 & 133796856.8 & 2.742154881 & 47.58 & 235728865.3 & 2.812657065 \\
\hline $\begin{array}{l}\text { Zeus } \\
\text { Linnaeus }\end{array}$ & $\begin{array}{l}\text { erMATOU } \\
\text { DAl }\end{array}$ & 4 & 0.002912522 & 0 & 0 & 0 & 0 & 0 & 0 & 0 & 0 & 4 & 0.00148711 & 1355504.447 & 1355.504447 & 0 & 0 & 0 & 0 & 0 & 0 \\
\hline 0 & 0 & 0 & 0 & 0 & 0 & 0 & 0 & 0 & 0 & 0 & 0 & 0 & 0 & 0 & 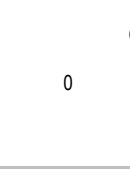 & $\begin{array}{c}\text { Estimated Total } \\
\text { Cs-134 in All Fish } \\
\text { in FUKUSHIMA- } \\
\text { KEN EEZ } \\
\text { [becquerel] = } \\
\text { [Estimated Total } \\
\text { Cs-134 in Fish] }\end{array}$ & 4879259657 & 0 & $\begin{array}{l}\text { Estimated Total } \\
\text { Cs-137 in All } \\
\text { Fish in } \\
\text { FUKUSHIMA- } \\
\text { KEN EEZ } \\
\text { [becquerel] }\end{array}$ & 8381002727 & 0 \\
\hline 0 & $\begin{array}{l}\text { Total fish } \\
\text { weight }\end{array}$ & h 137338 & 0 & 32960 & 0 & 37590 & 0 & 13130 & 0 & 47960 & 0 & 268978 & 0 & 0 & 91150218.79 & 0 & 0 & 0 & 0 & 0 & 0 \\
\hline
\end{tabular}


quantity of radioisotopes in fish biomass cover broad ranges extending from offshore to the boundaries of designated areas, such as EEZs (Moiseev and Kardashev, 1964).

\section{Conclusion}

(1) The estimated total fish biomass in the FukushimaKen EEZ on 22 to 23 November, 2012 was $91,150,218.79 \mathrm{~kg}$.

(2) The estimated total quantities of Cs-134 and Cs-137 in all fish in the Fukushima-Ken EEZ on 22 to 22 November, 2012 were 4,879,259,657 Bq and $8,381,002,727 \mathrm{~Bq}$, respectively.

\section{ACKNOWLEDGMENTS}

Hidemitsu KATSURA thanks Ms. ARIKI Mizuki, Ms. GORAI Myu, Ms. HENMI Chikako, Mr. KASE Ryota, Mr. NAKAGAWA Takuro and Mr. MYOUSE Hiroshi, undergraduate students at the Tokyo University of Marine Science and Technology (TUMST); Ms. SHIMOMURA Yukiko, Mr. HASEGAWA Kouhei, and Mr. WATANABE Hayato, graduate students at TUMST; Ph.D. Candidate GIRAULT Mathias and Ph.D. Candidate MATSUMOTO Akira at TUMST; Mr. SATO Katsuhiro and Ms. YOSHIZAKI Kae, Research Scientists, TUMST; Ms. HONDA Tomoko, Secretary to Professor ARAKAWA Hisayuki, TUMST; Dr. WAKABAYASHI Kaori, Doctoral Research Scientist, TUMST; Dr. NORMAN Chris, Adjunct Professor, TUMST; Dr. AKIYAMA Seiji, Assistant Professor, TUMST; Dr. UCHIDA Keiichi, Assistant Professor, TUMST; Dr. ARAKAWA Hisayuki, Professor, TUMST and Dr. MORINAGA Tsutomu, Emeritus Professor TUMST for their assistance. This study was supported by the Research Thrust Fund for fiscal year 2012 of the Ministry of the Environment, Japan for "Study of the diffusion process of radioisotope materials from the destroyed nuclear power plant in offshore ecosystems in Fukushima-Ken (Prefecture), Japan [HEISEI 24 NENDO KANKYOUSHOU SOUGOU KENKYUU SUISHINHI]".

\section{REFERENCES}

Asmolov VG, Borozhov AA, Demin VF, Kalugin AK, Kuz'min II, Kulakov VM, Legasov VA, Lunin GL, Ponomarev-Stepnoi NN (1987). The accident at the Chernobyl nuclear power plant: One year after. Energia Nucleare (Rome) 4(3):7-30.

Atkins WRG, Warren FJ (1953). The preservation of fishing nets, trawl twines, and fiber ropes for use in sea water. J. Mar. Biol. Assoc. United Kingdom 31:509-13.

Buscaino, G, Buffa G, Sara G, Bellante A, Tonello AJ Jr, Hardt FAS, Cremer MJ, Bonanno A, Cuttitta A, Mazzola S (2009). Pinger affects fish catch efficiency and damage to bottom gill nets related to bottlenose dolphins. Fisheries Sci. (Tokyo, Japan) 75(3):537-544.

Inazu T, Tsunoda A, Ohnishi S, Matsubara Y (2011). Determination of radioactive substances in foods by $\mathrm{Ge}$ semiconductor detector. Kenkyu Hokoku - Kagawa-ken Sangyo Gijutsu Senta 12:67-68.

Japanese Governmental Official Bulletin On-Line (2012). http://www.gov-online.go.jp/useful/article/201204/3.html.

Lown B, Pastore JO (1985). A medical prescription for survival. Lancet 2(8467):1285-1287

Macdonald HF, Harte GA, Corbett, JO (1987). Developments in emergency monitoring arrangements at Central Electricity Generating Board nuclear power stations since the Three Mile Island accident. Nuclear Energy (British Nuclear Energy Society) 26(1):31-40.

Minatani T, Nagai H, Nakamura M, Otsuka K, Sakai Y (2012). Radioactive cesium analysis in radiation-tainted beef by gamma-ray spectrometry with germanium semiconductor detector. Shokuhin eiseigaku zasshi. J. Food Hyg. Soc. Jap. 53(4):177-82.

Ministry of Education, C., S., S. \& T. (2011). Monitoring information of environmental radioactivity level, Reading of environmental radioactivity level by prefecture [Fallout], March、2011. Announce.: 1400 29-JUL-2011, Corrected: 28-SEP-2011, Add.: 14-DEC-2011, http://radioactivity.mext.go.jp/old/ja/monitoring_by_prefecture_fallout/ 2011/03/1060_03_gekkan_2.pdf.

Moiseev PA, Kardashev AV (1964). Radioactivity of certain marine life of the Pacific in 1958, following nuclear weapon tests carried out by the U.S.A. in the Central Pacific Ocean. Radioaktivn. Zagryaznennost Morei i Okeanov. Akad. Nauk SSSR, Okeanogr. Komis. 1964:126-35.

Nemoto Y, Shimamura S, Igarashi S (2012). Influence of radioactive substances on the marine organism and fishing areas off Fukushima Prefecture. Nippon Suisan Gakkaishi 78(3):514-519

Ohnishi T (2012). The disaster at Japan's Fukushima-Daiichi nuclear power plant after the March 11, 2011 earthquake and tsunami, and the resulting spread of radioisotope contamination. Rad. Res. 177(1):1-14.

Scherb H, Voigt K (2011). The human sex odds at birth after the atmospheric atomic bomb tests, after Chernobyl, and in the vicinity of nuclear facilities. Environ. Sci. Pollut. Res. Int. 18(5):697-707.

Wan M, Zal U, Yii M (2012). Marine radioactivity concentration in the Exclusive Economic Zone of Peninsular Malaysia: 226Ra, 228Ra and 228Ra/226Ra. J. Radioanalytical Nucl. Chem. 292(1):183-192. 